\title{
Formation of electron bunches for harmonic cascade x-ray free electron lasers
}

\author{
M. Cornacchia, S. Di Mitri, and G. Penco \\ Sincrotrone Trieste, Trieste, Italy \\ A. A. Zholents \\ Lawrence Berkeley National Laboratory, Berkeley, California 94720, USA
}

(Received 11 August 2006; published 6 December 2006)

\begin{abstract}
Specific requirements for the electron beam in harmonic cascade-free electron lasers, together with means to produce such beams, are presented. All results are illustrated with simulations and particle tracking studies.
\end{abstract}

DOI: 10.1103/PhysRevSTAB.9.120701

PACS numbers: 41.50.+h, 41.60.Cr, 42.55.Vc, 52.59.-f

\section{INTRODUCTION}

The operation of x-ray free electron lasers (FELs) relies on extremely high quality electron beams. Two FEL projects employing the technique of self-amplified spontaneous emission define the state-of-the-art situation: peak current of few kiloamperes, emittance of $1 \mathrm{~mm}$-mrad or less, and an energy spread of $1 \mathrm{MeV}$ or less [1,2]. Creation of electron bunches with these parameters is a difficult and elaborate process consisting of the electron bunch production, acceleration, and compression. A significant understanding was gained in the underlying physics over the past decade [3-7]. The main phenomena affecting electron bunches includes space charge effects, wakefields, and coherent synchrotron radiation (CSR). Nonlinearity of the waveform of the accelerating field in the linac and nonlinear time-of-flight characteristics of bunch compressors also play an important role.

More demanding for the electron beam quality are FELs that are designed to generate temporally coherent x-rays. These FELs, called high-gain harmonic generation FELs or harmonic cascade FELs (HC FELs) [8-10], employ a laser to seed the radiation at a lower harmonic of the output FEL radiation. Very often several FEL cascades are used to obtain the radiation at the $\mathrm{x}$-ray wavelength. In these cases, the radiation produced in one cascade by one group of electrons proceeds ahead and interacts with other electrons from the same electron bunch in the next cascade. Thus, relatively long electron bunches are needed to accommodate this technique. It is important to have a constant peak current (i.e. a flat electron density distribution) over the entire bunch length, and a discussion of the means to obtain this is one of the objectives of this paper. We also propose a technique aiming for control of the peak current spikes at the edges of the electron bunch. Those spikes often occur after the final bunch compression and are capable of inducing unwanted wakefields and coherent synchrotron radiation.

It is largely anticipated that HC FELs will be used for the production of relatively long temporally coherent x-ray pulses with a narrow bandwidth. However, a nonlinear energy modulation of electrons in the electron bunch can cause a frequency chirp in the output signal $[11,12]$ which broadens the bandwidth significantly beyond the Fourier transform limit. ${ }^{1}$ The rf harmonic linearizer [13] often helps to remove major nonlinear components in the energy modulation of electrons, but, frequently, even the remaining modulation significantly broadens the bandwidth, in particular, when strong linac structural wakefields are present. In this paper we propose a complementary technique which employs a specially shaped distribution of electron density and the wakefields themselves in order to avoid the remaining energy modulation of electrons. We also give a simple recipe on how to find such a distribution and demonstrate its usefulness with a practical example.

The overall goal for a design of the electron beam delivery system responsible for the formation of the electron bunches for HC FEL is to obtain a so-called flat-flat distribution, i.e., flat in the peak current and flat in the energy, which means that there are no peak current and energy modulations of electrons along the electron bunch. The technique discussed in this paper allows achievement of this goal on a macroscale comparable to the bunch length, but does not deal with the peak current and energy fluctuations on a microscale of few tens of microns often caused by the microbunching instability. This problem has already been addressed in the literature (see $[4,14]$ and references therein).

\section{REVERSE TRACKING}

The origins of the energy modulation of electrons appearing on a scale comparable to the electron bunch length include nonlinear time dependent variations of the rf fields, linac structural wakefields, and resistive wall wakefields. CSR also causes energy modulation on a scale comparable to the electron bunch length, but, typically, with smaller amplitude than that of other effects. A combined action of wake and rf fields plus bunch compression manipulations

\footnotetext{
${ }^{1}$ Bandwidth broadening can also be caused by a frequency chip in the seed laser.
} 
in the magnetic chicanes form the final electron beam distribution in the longitudinal phase space. We note that all these effects do not cause a mixture of electrons in the phase space and are reversible. Therefore, the basic philosophy that we follow in this paper is that the output bunch configuration is largely predetermined by the input bunch configuration and, thus, it is possible to find a unique electron density distribution at the beginning of the linac that produces a flat-flat distribution at the end of the linac. Finding this distribution can be relatively easy. One just needs to reverse the problem, i.e., start at the end of the linac and move backwards towards the beginning of the linac. Equation (1) shows that for a given normalized electron density distribution $\lambda$ and the point-charge wake function $w$, the relative electron energy $\delta=\left(E-E_{0}\right) / E_{0}$, where $E_{0}$ is the equilibrium energy, defined at the end of a section of the linac as $\delta_{f}$ can be determined using coordinate $s_{i}$ (measured with respect to the bunch center) and the electron energy $\delta_{i}$ at the beginning of this linac section:

$$
\begin{aligned}
\delta_{f}\left(s_{f}\right)= & \delta_{i}\left(s_{i}\right)+\frac{e U}{E_{0}}\left[\cos \left(k s_{i}+\phi\right)-\cos (\phi)\right] \\
& +\frac{e^{2} N L_{\mathrm{acc}}}{E_{0}} \int_{0}^{s_{i}} w\left(s_{i}-s^{\prime}\right) \lambda\left(s^{\prime}\right) d s^{\prime} .
\end{aligned}
$$

Here $U, \phi, L_{\text {acc }}$ are the voltage, phase, and length of the linac section, $k$ is the $\mathrm{rf}$ wave number, $e$ is the electron charge, and $N$ is the number of electrons in the bunch. In a relativistic beam the electron distribution function $\lambda$ does not change during acceleration and the electron coordinate at the end of the linac $s_{f}$ remains the same as that at the beginning of the linac, i.e. $s_{f} \equiv s_{i}$. Therefore, Eq. (1) can be used to define $\delta_{i}\left(s_{i}\right)$ as a function of $\delta_{f}\left(s_{f}=s_{i}\right)$. Thus, beginning with a desirable electron distribution at the end of the linac, one can find the distribution at the start that, once tracked forward, will reproduce the desired distribution at the end of the linac.

A different situation arises in a bunch compressor where the electron coordinate at the end of the bunch compressor $s_{f}\left(\delta_{f}\right)$ becomes a function of the electron coordinates $s_{i}$ and energy $\delta_{i}$ at the beginning of the bunch compressor and where electron energy losses occurred during passage through the bunch compressor:

$$
\begin{aligned}
s_{f}\left(\delta_{f}\right)= & s_{i}\left(\delta_{i}\right)+R_{56} \delta_{i}+T_{566} \delta_{i}^{2}+f_{\mathrm{CSR}}\left(s_{i}\right) \\
& +f_{\mathrm{RW}}\left(s_{i}\right) \ldots,
\end{aligned}
$$

where $R_{56}, T_{566}$ are first and second order time-of-flight parameters and the functions $f_{\mathrm{CSR}}$ and $f_{\mathrm{RW}}$ describe changes in the electron coordinate caused by electron energy losses occurred inside the bunch compressor due to the CSR and resistive wall wakefields. However, in the case of a smooth electron density distribution, the emission of synchrotron radiation is coherent at frequencies $\omega \leq$ $c / \ell_{b}$, where $c$ is the speed of light and $\ell_{b}$ is the characteristic length of the scale of the bunch length [15]. Because the electron bunch moves inside the vacuum chamber, most of the spectral components of the CSR do not propagate if $\ell_{b}$ is relatively long, as it is often the case for HC FEL. Therefore, the loss of electron energy due to CSR is often much smaller than otherwise expected in a free space environment (see Fig. 1). As for the energy losses due to the resistive wall wakefields, they are also weak inside the bunch compressor or can be made weak by a proper choice of the aperture of the vacuum chamber.

Thus, if one ignores CSR and resistive wall wakefield and assumes that the electron energy is not affected in the bunch compressor, i.e. $f_{\mathrm{CSR}}=f_{\mathrm{RW}} \equiv 0$ and $\delta_{f} \equiv \delta_{i}$, then the electron coordinate at the beginning of the bunch compressor can be found using the electron coordinate at the end of the bunch compressor using Eq. (2).

The above considerations justify a concept of reverse tracking demonstrated in Fig. 2 using plots of the longitudinal phase space and the electron peak current. Figure 2(a) shows the result of conventional forward particle tracking obtained with LITRACK [17] starting with the electron distribution at the beginning of the linac at approximately $100 \mathrm{MeV}$ beam energy where the electrons are already sufficiently relativistic and Fig. 2(b) shows the reverse tracking starting with the distribution obtained at the end of the first tracking [see bottom part of Fig. 2(a) and top part of Fig. 2(b)]. The result of this tracking [bottom part of Fig. 2(b)] agrees well with the initial distribution [top part of Fig. 2(a)].

In the next step, a desirable flat-flat distribution was set up at the end of the accelerator. Starting with this distribution and tracking it backward, the nearly linear ramped peak current shown in Fig. 3 was obtained at the starting point. This result can be understood if one uses the wake function for an accelerating section consisting of an array of cells [18]:

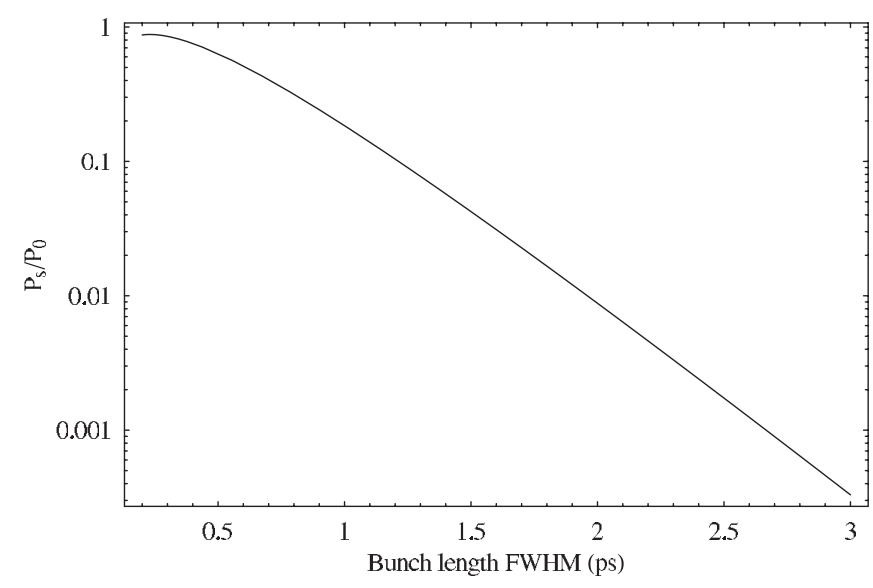

FIG. 1. Suppression of CSR due to the shielding effect from the vacuum chamber with a gap of $8 \mathrm{~mm}$. This result was obtained by following the recipe proposed in [16]. 

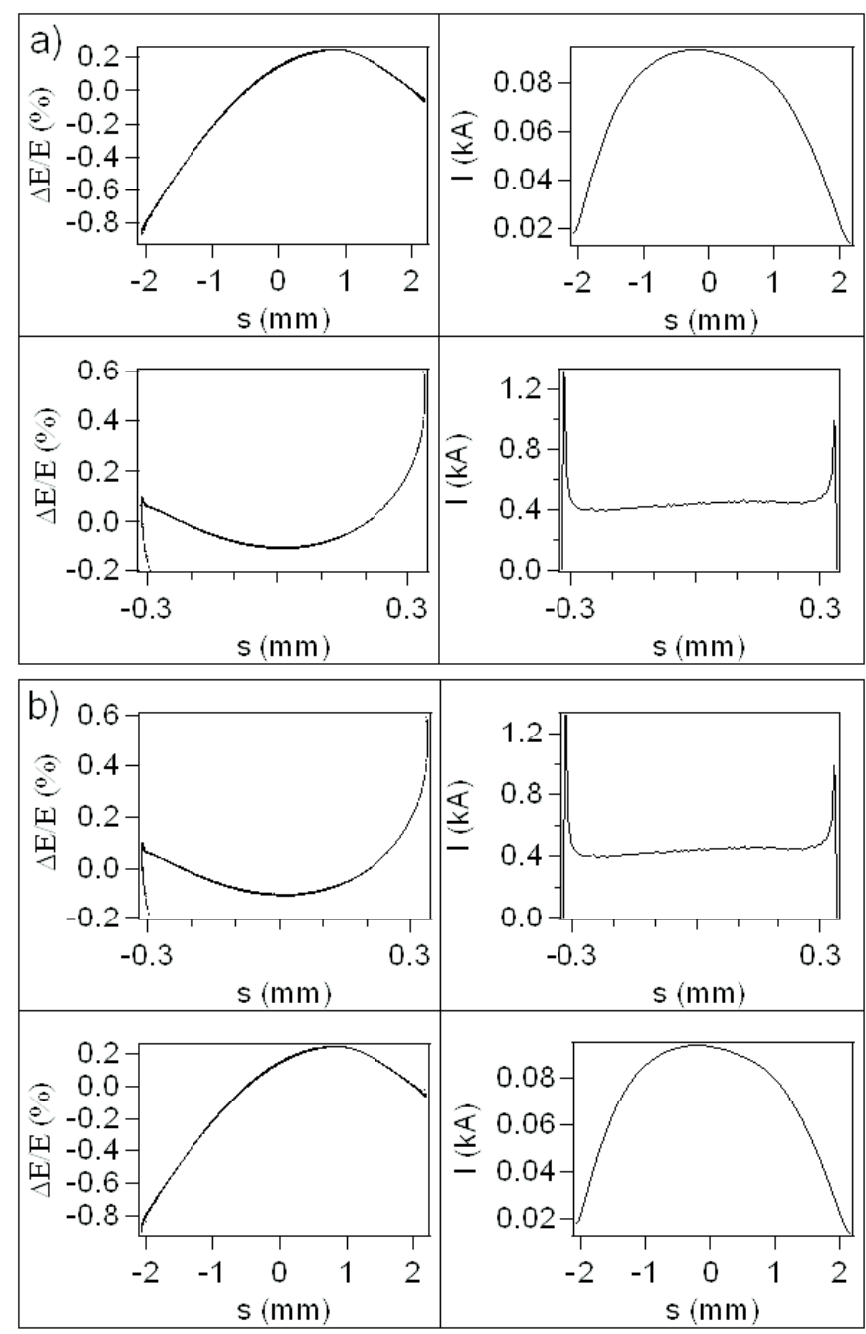

FIG. 2. Plot (a) shows forward particle tracking and plot (b) shows reverse particle tracking. Here and through the rest of the paper, the head electrons have negative $s$.

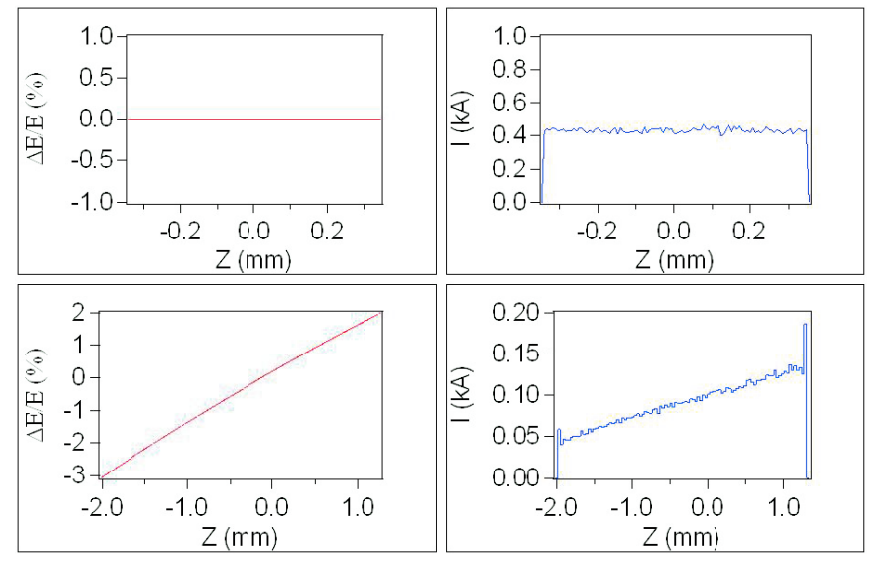

FIG. 3. (Color) Reverse tracking beginning with flat-flat distribution at the end of the accelerator (top) and moving towards beginning of the accelerator (bottom).

$$
w(s)=-A \frac{Z_{0} c}{\pi a^{2}} \exp \left(-\sqrt{s / s_{0}}\right)
$$

and convolute it with the linear ramped peak current distribution shown with the red line in Fig. 4(a) to obtain the wake potential

$$
W(s)=L_{\mathrm{acc}} \int_{-\infty}^{s} w\left(s-s^{\prime}\right) \lambda\left(s^{\prime}\right) d s^{\prime}
$$

shown with the red line in Fig. 4(b). Here $a$ is the iris radius, $Z_{0}=377 \Omega$ and $A \approx 1$ and $s_{0}$ are fitting coefficients. As seen in Fig. 4(b), the wake potential is highly linear and this is why the final distribution is flat in energy.

One can also consider applying the above described technique in a case when all or some of the adapted constraints such as weak CSR or wakefields in the bunch compressors are not completely met. In this case a backward tracking without CSR and wakefields can provide a good starting point for iterative processes in a search of a proper distribution.

Producing the linear ramped peak current distribution at the beginning of the linac is a challenging task addressed in [19]. The main idea is to use the photocathode laser and to shape the intensity of its pulse such as to produce the electron density distribution at the cathode that will eventually evolve due to the space charge forces into the linear ramped peak current distribution at the end of the injector (assumed to be at approximately $100 \mathrm{MeV}$ ). Simulations show the best distribution (shown with the red curve in Fig. 5) has a characteristic quadratic ramp in the electron density. This distribution evolves into the one shown with the black line in Fig. 4(a), where the linear ramp in the electron peak current occupies a larger fraction compared to all other tried cases.

Now consider a condition that allows a conversion of a linear ramped peak current at the beginning of the accelerator into the flat distribution at the end of the compression. A compression factor can be defined as
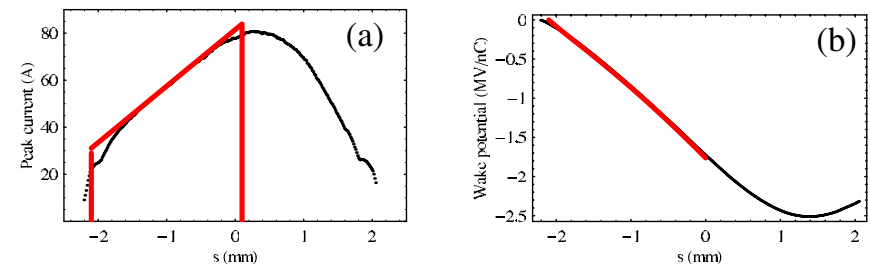

FIG. 4. (Color) (a) Electron distribution with a linear ramped peak current and (b) a correspondent wake potential calculated for $a=9.73 \mathrm{~mm}, s_{0}=1.28 \mathrm{~mm}$, and $L_{\text {acc }}=24 \mathrm{~m}$. The red line shows a desirable ideal distribution and its associated wake potential. The black line shows the realistic electron density distribution obtained in the photoinjector using the intensity shaped laser pulse (a), and the wake potential that corresponds to that distribution (b). The part under the red line contains approximately $40 \%$ of the total charge containing under the black line. 


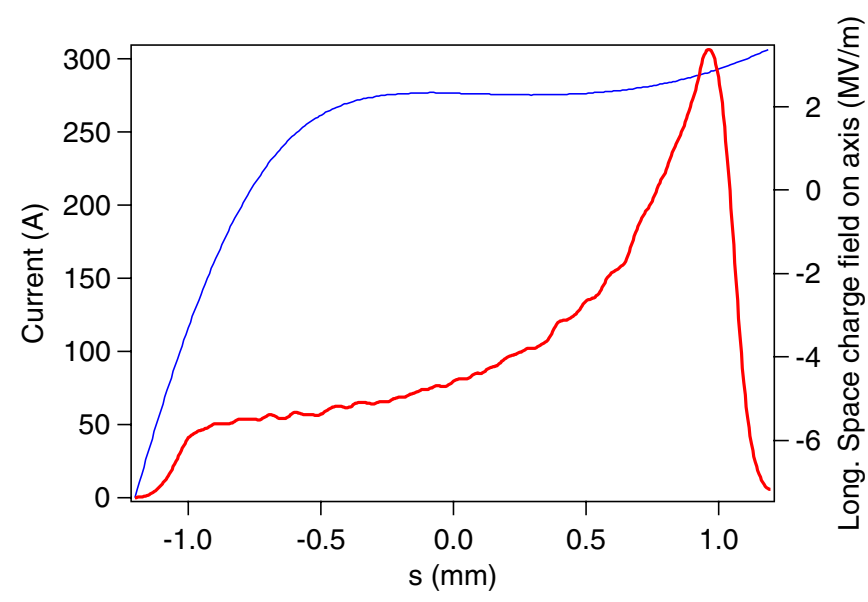

FIG. 5. (Color) The electron density distribution at the cathode (red curve) that evolves into the distribution with a linear ramped peak current shown in Fig. 4(a) with the black curve. The on-axis longitudinal space charge field (blue curve) of this distribution has a significant plateau part.

$$
C=I_{a} / I_{b},
$$

where $I_{a}$ is the peak current after compression and $I_{b}$ is the peak current before compression. A transformation from a linear ramped peak current $I_{b}=I_{b 0}+I_{b}^{\prime} s$ to the flat distribution $I_{a}=I_{a 0}=$ const can be obtained with

$$
C^{-1}=I_{b 0} / I_{a 0}+s I_{b}^{\prime} / I_{a 0}
$$

i.e. with a compression factor that gradually decreases from the head to the tail of the electron bunch. On the other side, the inverse of the compression factor is conventionally defined as $C^{-1}=1+h R_{56}$, where $h=$ $d(\Delta E / E) / d s$ is the energy chirp in the electron bunch. Thus, Eq. (6) can be satisfied if one uses the energy chirp with a quadratic component, i.e. $h=h_{0}+h^{\prime} s$, where

$$
h^{\prime}=\frac{1}{R_{56}} \frac{I_{b}^{\prime}}{I_{a 0}} .
$$

This result can be easily generalized. For example, in some cases a distribution with the maximum peak current at the head of the bunch gradually tapering off towards the tail can be beneficial for HC FEL, i.e., one may want $I_{a}=$ $I_{a 0}-I_{a}^{\prime} s$. Performing a similar analysis, one can find that this distribution can be obtained with a slight modification to the quadratic energy chirp:

$$
h^{\prime}=\frac{1}{R_{56}}\left(\frac{I_{b}^{\prime}}{I_{a 0}}+\frac{I_{a}^{\prime} I_{b 0}}{I_{a 0}^{2}}\right)
$$

\section{BIFURCATION IN PHASE SPACE AND PEAK CURRENT SPIKES}

The peak current spikes at the edges of the compressed electron bunches are largely due to the compressor's sec-

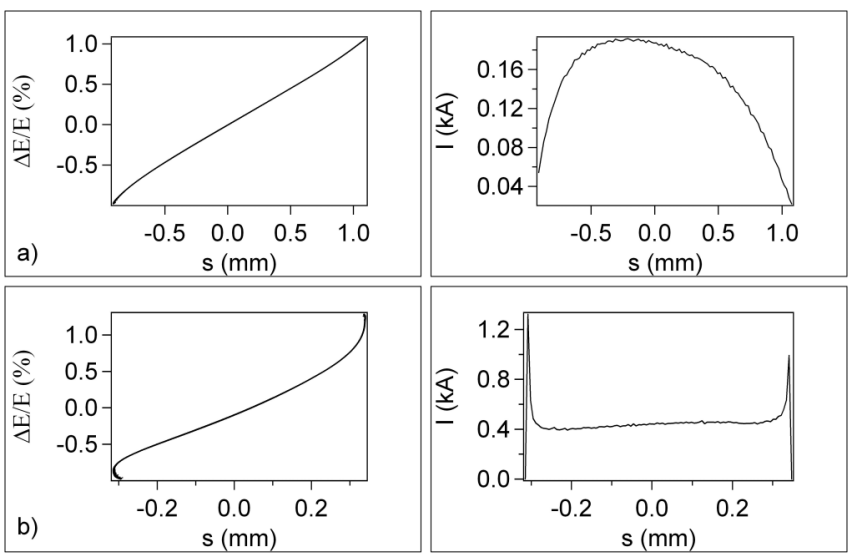

FIG. 6. Electron distribution in the longitudinal phase space: (a) before bunch compressor, (b) after the bunch compressor.

ond order time-of-flight parameter $T_{566}$ and the cubic chirp in the electron energy distribution $\mu=d^{3} E / d s^{3}$. Both of them cause the bifurcation in the phase space whose example is shown in Fig. 6(b). Controlling $\mu$ is likely the only way to control the bifurcation for a given $R_{56}$ since $T_{566}$ is often bound to $T_{566} \approx-3 / 2 R_{56}$ if sextupoles are not used in the bunch compressor.

Figure 7 shows a typical example that demonstrates how the change in $\mu$ from $-0.008 \mathrm{MeV} / \mathrm{mm}^{3}$ to $-0.088 \mathrm{MeV} / \mathrm{mm}^{3}$ in the electron energy distribution created in the injector removes the bifurcation after the bunch compressor. Related to that is a drop in the spikes in the peak current from $\sim 5 \mathrm{kA}$ in the first case to approximately a no spikes condition in the second case. Apart from the cubic energy chirp, the same initial distributions were used in both cases.

It should be pointed out that the distribution in Fig. 7(a) is "flat" in energy and in peak current, while the distribution in Fig. 6(b) is neither flat in the energy nor is it flat in the peak current. By using a cubic energy chirp as a knob,
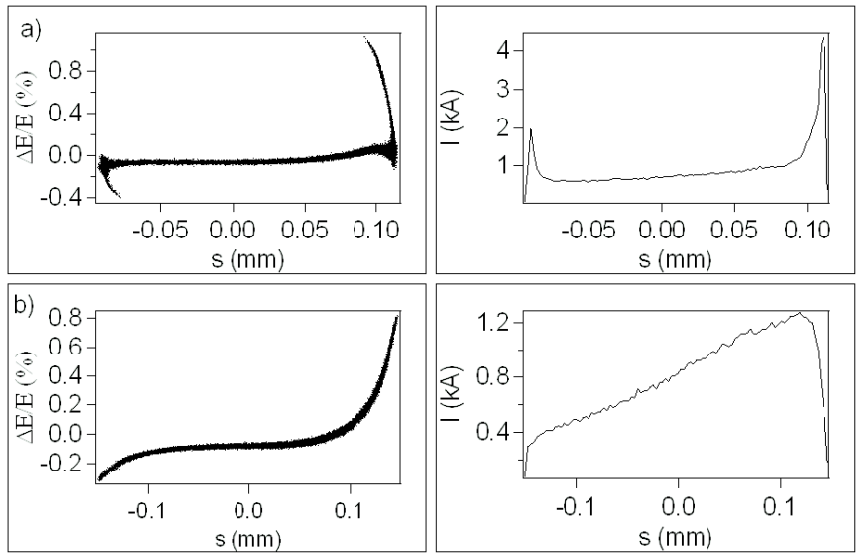

FIG. 7. Longitudinal phase space and peak current distributions: (a) $\mu=-0.008 \mathrm{MeV} / \mathrm{mm}^{3}$, (b) $\mu=$ $-0.088 \mathrm{MeV} / \mathrm{mm}^{3}$. 


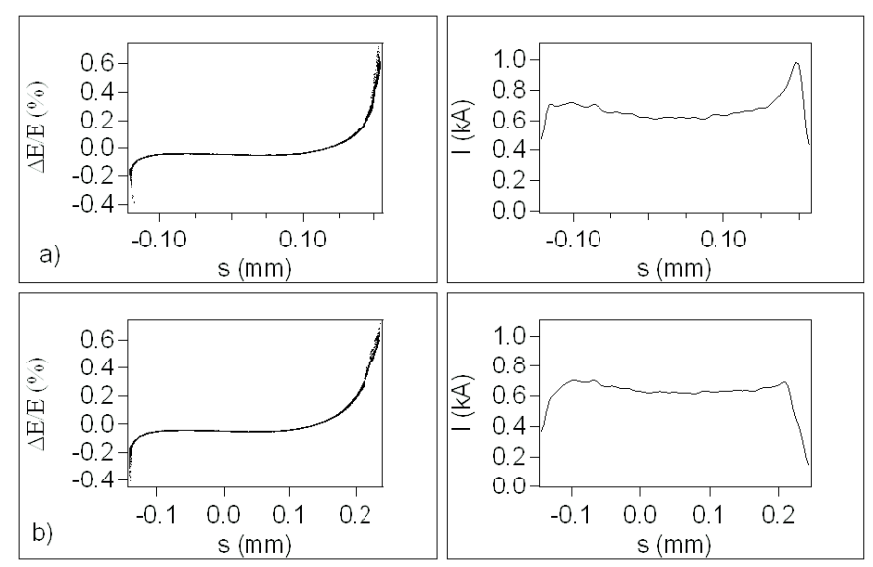

FIG. 8. The illustration for the removal of the spikes in the peak current at the electron bunch edges using adjustment in the $R_{56}$ of "dog-leg" lattice: (a) $R_{56}=2.8 \mathrm{~mm}$, (b) $R_{56}=7 \mathrm{~mm}$.
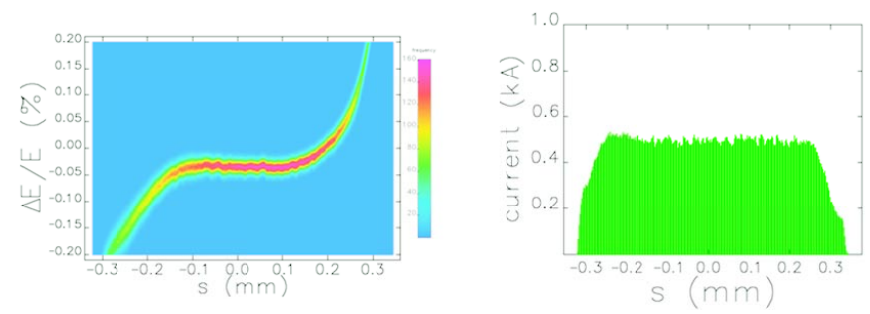

FIG. 9. (Color) The electron density distribution in the longitudinal phase space (left plot) and a histogram of the peak current (right plot) at the entrance of the FEL undulators calculated for the FERMI@Elettra linac.

one can obtain either flat-flat distributions or distributions without spikes rather routinely, but not both features at the same time. Simply having just one knob is not enough. However, one can effectively employ the peak current distribution in the gun to provide the needed $\mu$ using the wakefields. In the case of strong wakefields this method was found to be much more effective than a correction using high harmonic cavity [20]. This approach already gave good results in the case of the distribution shown with the black line in Fig. 4(a).

The electrons sitting at the edges of the electron bunch often deviate in energy from the energy of most other electrons. This feature can be explored in a complementary procedure for the removal of the spikes in the peak current if the electron distribution in the phase space has a characteristic s-type shape, like one shown in Fig. 8. (We note that the s-shaped distribution in the longitudinal phase space at the end of the linac appears rather naturally when ramped peak current in the injector is used.) Then, it is possible to dissolve clusters of electrons at the edges of the electron bunch by sending the electron beam through the lattice with a properly adjusted time-of-flight parameter $R_{56}$. Typically a "dog-leg" type lattice is used to connect the end of the linac to a chain of FEL's undulators and the necessary adjustments in $R_{56}$ can be easily done there. The example of the application of this technique is shown in Fig. 8.

The particle tracking code ELEGANT [21] was used in order to obtain the electron distribution in the longitudinal phase space and a histogram of the electron peak current at the entrance of the FEL, thus verifying the validity of all the techniques described here. These calculations were performed for FERMI@Elettra linac [22] using a ramped peak current distribution at the entrance of the linac shown with the black line in Fig. 4(a). The result of these calculations is shown in Fig. 9. We note that these calculations included CSR effects in the bunch compressors, but predictably its impact is not much visible on a large time scale.

\section{CONCLUSION}

In the presence of wakefields, the electron density distribution plays an important role in the formation of electron bunches at the end of the accelerator. Often the capability to manipulate the electron density during acceleration and compression is limited and cannot provide the wanted results. However, use of the laser in the photocathode gun opens a new opportunity to affect the electron density distribution by controlling its intensity. This paper provides a practical example where the electron density distribution with a linear ramped peak current, obtained through a quadratic ramp in the laser intensity, had many useful implications.

\section{ACKNOWLEDGMENTS}

We are grateful to P. Emma, G. Stupakov, and C. Pellegrini for many useful discussions. In part this work was supported by U.S. Department of Energy under Contract No. DE-AC02-05CH11231.

[1] LCLS CDR, SLAC Report No. SLAC-R-593, 2002.

[2] TESLA TDR, DESY Report No. DESY-2001-011, 2001.

[3] M. Borland, Y. C. Chae, P. Emma, J. W. Lewellen, V. Bharadwaj, W. M. Fawley, P. Krejcik, S. V. Milton, H. Nuhn, R. Soliday, and M. Woodley, Nucl. Instrum. Methods Phys. Res., Sect. A 483, 268 (2002).

[4] E. L. Saldin, E. A. Schneidmiller, and M. V. Yurkov, Nucl. Instrum. Methods Phys. Res., Sect. A 490, 1 (2002).

[5] S. Heifets, G. Stupakov, and S. Krinsky, Phys. Rev. ST Accel. Beams 5, 064401 (2002).

[6] Z. Huang and K.-J. Kim, Phys. Rev. ST Accel. Beams 5, 074401 (2002).

[7] K. Bane, SLAC-PUB-11829, 2006.

[8] R. Bonifacio, L. De Salvo Souza, and E. T. Scharlemann, Nucl. Instrum. Methods Phys. Res., Sect. A 293, 627 (1990).

[9] L.-H. Yu et al., Science 289, 932 (2000).

[10] G. Penn, M. Reinsch, J. Wurtele, J. N. Corlett, W. M. Fawley, A. Zholents, and W. Wan, "Harmonic Cascade 
FEL Designs for LUX," Proceedings of European Particle Accelerator Conference 2004, Lucerne.

[11] S. G. Biedron, S. V. Milton, and H. P. Freund, Nucl. Instrum. Methods Phys. Res., Sect. A 475, 401 (2001).

[12] T. Shaftan and L. H. Yu, Phys. Rev. E 71, 046501 (2005).

[13] D. H. Dowell, T. D. Hayward, and A. M. Vetter, "Magnetic Pulse Compression Using a Third Harmonic RF Linearizer," Proceedings of the 1995 Particle Accelerator Conference 1995, New York.

[14] Z. Huang, M. Borland, P. Emma, J. Wu, C. Limborg, G. Stupakov, and J. Welch, Phys. Rev. ST Accel. Beams 7, 074401 (2004).

[15] L. I. Schiff, Rev. Sci. Instrum. 17, 6 (1946).

[16] R. Li, C. L. Bohn, and J. J. Bisognano, "Analysis of the Steady-State Coherent Synchrotron Radiation with Strong Shielding," Proceedings of the Particle Accelerator Conference 1997, Vancouver, Canada, pp. 1644-1646.

[17] K. L. F. Bane and P. Emma, "LiTrack: A Fast Longitudinal Phase Space Tracking Code with Graphical User
Interface," Proceedings of the Particle Accelerator Conference 2005, Knoxville, Tennessee, pp. 4266-4268.

[18] K. Bane, A. Mosnier, A. Novokhatski, and K. Yokoya, "Calculations of the Short-Range Longitudinal Wakefields in the NLC Linac," Proceedings of the ICAP Conference 1998, Monterey, California.

[19] G. Penco, M. Trov'o, and S. Lidia, "Ramping Longitudinal Distribution Studies for the Fermi@Elettra Injector," Proceedings of the FEL Conference 2006, Berlin, Germany.

[20] K. Flottmann, T. Limberg, and Ph. Piot, DESY-TESLAFEL-2001-06.

[21] M. Borland, APS Tech Note LS-207, 2000.

[22] M. Cornacchia, P. Craievich, S. Di Mitri, I. Pogorelov, J. Qiang, M. Venturini, A. Zholents, D. Wang, and R. Warnock, "Study of the Electron Beam Dynamics in the FERMI@Elettra Linac," Proceedings of European Particle Accelerator Conference 2006, Edinburgh, UK, pp. 145-147. 